\title{
The Role of Stem Cell Genomic Instability in Aging
}

\author{
Cynthia J. Hommerding ${ }^{1}$ - Bennett G. Childs ${ }^{1}$ - Darren J. Baker ${ }^{1,2}$
}

Published online: 26 June 2015

(C) Springer International Publishing AG 2015

\begin{abstract}
Organismal aging is characterized by a progressive loss of tissue homeostasis and impaired function over time. Multi-cellular organisms activate stem and progenitor cells to replace damaged cells in order to continuously meet the functional demands of tissues. Along with tissue dysfunction, stem cell self-renewal and differentiation capacities diminish with age in concert with the accumulation of genomic damage, suggesting a potential link between genetic instability and aging. Here, we focus on the types of DNA damage found in aged stem cells, and discuss emerging mechanisms by which genomic instability may contribute to stem cell impairments and organismal aging, with particular emphasis on insights obtained from progeroid mouse models. Additionally, we discuss how age-related systemic changes may impact stem cell genomic integrity and function.
\end{abstract}

Keywords Aging $\cdot$ Genomic instability $\cdot$ DNA damage $\cdot$ Stem cells $\cdot$ Progeroid

This article is part of the Topical Collection on Age-related Stem Cell Modifiers

Cynthia J. Hommerding and Bennett G. Childs contributed equally to this work.

Darren J. Baker

baker.darren@mayo.edu

1 Department of Biochemistry and Molecular Biology, Mayo Clinic, 200 First St. SW, Rochester, MN 55905, USA

2 Department of Pediatric and Adolescent Medicine, Mayo Clinic, 200 First St. SW, Rochester, MN 55905, USA

\section{Introduction}

One inevitable consequence of aging is diminished health. Over time, there is accumulating macromolecular damage to proteins and/or DNA due to defective repair, reduced dilution/ degradation of protein aggregates, and increased free radical production. DNA acquires mutations from both intrinsic (i.e., defective DNA duplication or oxidation) and extrinsic (i.e., UV damage, ionizing radiation, exposure to chemicals) factors. Upon DNA damage, eukaryotic cells initiate stress responses that are unique to the type and extent of damage, and ultimately determine cell fate [1]. In response to damage, cells will generally undergo a transient arrest in order to repair the damage before re-entering the cell cycle [2, 3]. However, if this arrest is insufficient to mediate effective repair, cells will either senesce (enter an irreversible cell cycle arrest) or undergo apoptosis [2,3]. Deficiencies in genome maintenance pathways have been observed with aging under normal conditions [4]. Consistent with this, unrepaired and/or persistent DNA damage has been linked to several diseases, including cancer and premature aging $[5,6]$.

Tissue homeostasis depends on stem cells that are functioning properly within tissues. These undifferentiated cells have several unique properties, including self-renewal and production of tissue-specific progenitor cells [7, 8]. In response to tissue damage, adult stem cells are activated to replace dysfunctional cells through the production of progenitor cells capable of differentiating into the cell types required to maintain normal tissue function [8]. If these stem and/or progenitor cells function suboptimally, this process is attenuated and may accelerate the onset of tissue aging [9]. Genomic damage may impede stem cell function at multiple levels, by causing cell death or withdrawal from the cell cycle, impairing differentiation or the self-renewal process, or through disruption of the stem cell microenvironment [10]. Here, we discuss the 
existence and significance of accumulated genomic damage to stem cell functionality during the aging process, with particular emphasis placed on pathways implicated and insights gained from progeroid mouse models, where genetic instability is promoted due to insufficient DNA damage responses.

\section{Age-Related Genomic Alterations}

Genomic damage occurs through a wide range of mechanisms and has been implicated or characterized in association with aged cells. These include, but are not limited to, impaired maintenance of telomeres, defective DNA repair in response to genotoxic and replication stress, epigenetic alterations, and genetic instability from translocations and missegregation of whole chromosomes [10]. Several of these defects are also observed frequently in cancer; however, for the purposes of this review, we limit our discussion to organismal aging.

Telomere integrity is important for cellular longevity, as tandem repeats at chromosome ends are lost continually during progressive rounds of DNA replication $[11,12]$. Once a critical telomere length threshold has been breached, the cell activates a robust DNA damage response. To prevent attrition, telomerase, a specialized DNA polymerase, has the capability of maintaining sufficient telomere lengths. However, most mammalian somatic cells and adult stem cells express very low amounts of telomerase $[13,14]$. Therefore, it is not surprising that telomere shortening is observed in both mice and humans with normal aging $[15,16]$. Most of our understanding of the significance of defective telomeres to genomic stability and aging come from cell culture studies [17-19] and specific disease pathologies in humans, including pulmonary fibrosis, dyskeratosis congenital, and aplastic anemia [20]. In addition, breeding of telomerase component null mice $\left(\mathrm{Terc}^{-/-}\right.$) for successive generations critically shortens telomeres, resulting in a shortened lifespan, intestinal and testicular atrophy, and increased incidence of lymphoma [21,22].

Genotoxic stress, either through endogenous or exogenous mechanisms, also contributes to disease and tissue/organ functional decline [10]. Endogenous stress, which occurs as a consequence of normal metabolism and cellular processes, includes hydrolysis or oxidation, resulting in depurination, deamination, and numerous other chemical lesions [23]. Exogenous stressors include radiation, such as UV light and ionizing radiation, and a number of environmental agents, such as benzo $[a]$ pyrene, which is present in cigarette smoke and diesel exhaust [23]. Exposure to these types of stresses will lead to the accumulation of DNA damage, if not immediately and effectively repaired. Cells within highly proliferative organs may accumulate mutations at a high rate during DNA replication, especially if the DNA repair machinery is defective $[10$, 23]. For example, normal human colon crypts acquire large chromosomal deletions, duplications, and gene conversions with age [24•], potentially due to loss of apoptotic potential. Mutations in numerous DNA repair genes have been linked to premature aging diseases in humans, including Cockayne syndrome, trichothiodystrophy, and ataxia telangiectasia. These diseases are characterized by cancer susceptibility and a number of progeroid phenotypes, including cachexia, kyphosis, retinal degeneration, and shortened lifespan [5]. Cockayne syndrome and trichothiodystrophy are caused by mutations to one of several genes associated with transcription-coupled repair (CSA, CSB, XPB, XPD, and TTDA) [5]. Ataxia telangiectasia is caused by mutations in the ataxia telangiectasia mutated gene (ATM), which functions as part of the DNA damage response (DDR), which is triggered by DNA double-strand breaks (DSBs) [25].

A number of epigenetic changes also occur throughout life to regulate gene expression and promote variation between different cell types [26]. Several histone marks, such as increased H4K16 acetylation, H4K20 or H3K4 trimethylation, and decreased $\mathrm{H} 3 \mathrm{~K} 9$ methylation or $\mathrm{H} 3 \mathrm{~K} 27$ trimethylation, have been associated with organismal aging [27-31]. Both a global loss of DNA methylation and hypermethylation of a subset of loci have also been described with progressive aging, and may result in silencing of active genes, activation of silenced genes, and accumulation of DNA damage due to open chromatin confirmation [32]. In addition, mice harboring mutations in the $L s h$ gene, which encodes PASG, a facilitator of DNA methylation, results in global hypomethylation, developmental growth retardation, and numerous premature aging phenotypes, including hair graying and loss, reduced fat deposition in skin, osteoporosis, kyphosis, cachexia, and a shortened lifespan [33]. Fibroblasts isolated from Lsh mutant mice exhibit premature replicative senescence in culture due to increased $\mathrm{p} 16^{\mathrm{INK} 4 \mathrm{~A}}$ expression, resulting from downregulation of Bmil, a negative regulator of $\mathrm{p} 16^{\mathrm{INK} 4 \mathrm{~A}}$ [33].

Aneuploidy, the state of having non-modal numbers of whole chromosomes, is caused by defective chromosomal segregation and has been detected in various tissues from wild-type mice with increasing age [34••]. In addition, premature aging and aneuploidy have been observed in mouse models with reduced levels of the mitotic checkpoint proteins BubR1, Bub3, and Rae1 [35, 36]. Heterozygous deletion of Bub3 or Rae1 promotes aneuploidy, but is insufficient to promote premature aging phenotypes in mice. However, haploinsufficiency at both loci causes significantly higher levels of aneuploidy than each model individually and promotes an earlier onset of age-related pathologies than wild-type mice [36]. Mice with reduced levels of BubR1 have an even earlier onset of a variety of progeroid phenotypes, including shortened lifespan, cachetic dwarfism, lordokyphosis, cataracts, loss of subcutaneous fat, impaired wound healing, and progressive aneuploidy [35]. Mutations in human $B U B 1 B$ have also been found in mosaic variegated aneuploidy (MVA) patients [37], a syndrome characterized by rampant aneuploidy. In addition, 
these patients develop a variety of phenotypes at a very young age, including short stature, facial abnormalities, and cataracts [38]. Taken together, these results demonstrate that aging is accompanied by a number of genetic insults. Their significance, however, is difficult to evaluate in the context of normal aging.

\section{Phenotypic Effects of DNA Damage in Stem and Progenitor Cells}

The majority of our understanding of how defective DNA damage repair impacts stem and progenitor cell function comes from mouse models (Table 1). For example, short, uncapped or dysfunctional telomeres elicit a DNA damage response. Mice with defective telomere maintenance due to deficiencies in Terc, the RNA template required for telomere replication, are prone to premature aging, including short lifespans, reduced body weight, intestinal and testicular atrophy, and increased incidence of lymphoma [21]. In order to observe these effects and because mouse telomeres are extremely long, Terc knockout mice require successive generations of breeding to develop critically shortened telomeres, which usually happens in the fifth or sixth generation. Importantly, $\operatorname{Terc}^{-/-}$mice exhibit defective hematopoietic stem cell (HSC) renewal, though it is unclear whether and how this contributes to the aging of this model. It is also unknown whether other stem cell pools are similarly impacted. When combined with loss of Atm, there is an even further reduction of HSC renewal and increased rates of apoptosis in neural stem cells and intestinal crypts [58]. Interestingly, mice that overexpress telomerase reverse transcriptase (TERT), the enzymatic component of telomerase, exhibit lengthened telomeres in hair bulge stem cells, improved fitness, and prolonged lifespan compared to wild-type controls [61]. This suggests that either mouse telomeres experience progressive shortening with age, which contributes to the development of age-related phenotypes once a critical threshold length is reached, or that TERT is providing another protective function. Without additional studies, neither possibility can be ruled out.

Deficient DNA DSB repair drives early aging in a number of genetically manipulated mice. Ataxia telangiectasia mutated (Atm) is a kinase rapidly recruited to DSBs by the Mre11/ Rad50/Nbs1 (MRN) complex to hold together the two DNA ends following damage [62]. In humans, mutations in this gene cause ataxia telangiectasia, a rare syndrome characterized by genetic instability, ataxia, slow growth, premature aging in skin and hair, radiation sensitivity, and a predisposition to tumorigenesis [63]. $\mathrm{Atm}^{-/-}$mice recapitulate many of these features [43] and also have abnormal HSC self-renewal [44]. Consistent with these findings, mice hypomorphic for $\operatorname{Rad} 50\left(\operatorname{Rad} 50^{\mathrm{S} / \mathrm{S}}\right)$, one of the proteins of the MRN complex, also have a short lifespan and HSC failure [48]. HSCs in wildtype mice also exhibit an increase in single- and double-strand breaks and cell cycle defects with age [64••, 65••], which are thought to accumulate, at least in part, through decreased expression of mini-chromosome maintenance (MCM) helicase components and altered replication fork dynamics [65••]. Additionally, high rates of DSBs are observed in HSCs of mice lacking FancA, a member of the Fanconi anemia complementation family of proteins important for DNA cross-link repair, following repeated infection and activation that ultimately leads to HSC failure [53••]. Together, these results indicate that defective DSB repair may commonly lead to depletion or dysfunction of HSCs. Stem cells of other tissues may also be impacted by similar mechanisms, although this remains unclear.

Unlike the previously mentioned models of premature aging, patients with Hutchinson-Gilford progeria syndrome (HGPS), a rare disorder characterized by limited growth, hair loss, and frailty reminiscent of advanced age, is not caused by defective DNA damage repair. This disease is caused by a spontaneous mutation in lamin A (LMNA), a scaffold component of the nucleus [66]. When mutated, this causes defects in nuclear structure, which is linked to epigenetic regulation alterations and loss of genetic material. Mouse models with inducible expression of mutant Lmna exhibit growth retardation, along with skin abnormalities and hair loss [56], which are linked to stem cell dysfunction in the skin due to the induction of senescence [57]. It is unclear in this case, however, if the stem cell phenotype or induction of senescence is related to changes in gene regulation or genomic instability, or both. It is likely, however, that senescence may be induced in these cells due to activation of cellular stress responses.

\section{Effects of Aneuploidy on Stem Cell Function}

Down's syndrome (DS) typically occurs due to nondisjunction of the maternal copy of chromosome 21 and is one of the most commonly observed chromosomal abnormalities in humans. This stable aneuploidy occurs in all cells of the body and results in profound phenotypic consequences, including slowed growth, a characteristic facial appearance, and mild to moderate intellectual impairment [67]. The Ts65Dn mouse model for DS, which is trisomic for 132 genes that are homologous to human genes found on chromosome 21 [41], exhibits defective self-renewal, proliferation, and differentiation of hematopoietic stem and progenitor cells, and a reduction in neural progenitors, which is at least partly through increased amounts of Usp16 [42•]. The defects in these stem and progenitor cell pools may explain the increased prevalence of red cell macrocytosis, B- and T lymphocyte abnormalities, thrombocytopenia, neutropenia, myelodysplasia, bone marrow failure, and neurologic deficiencies observed in DS patients [67]. 


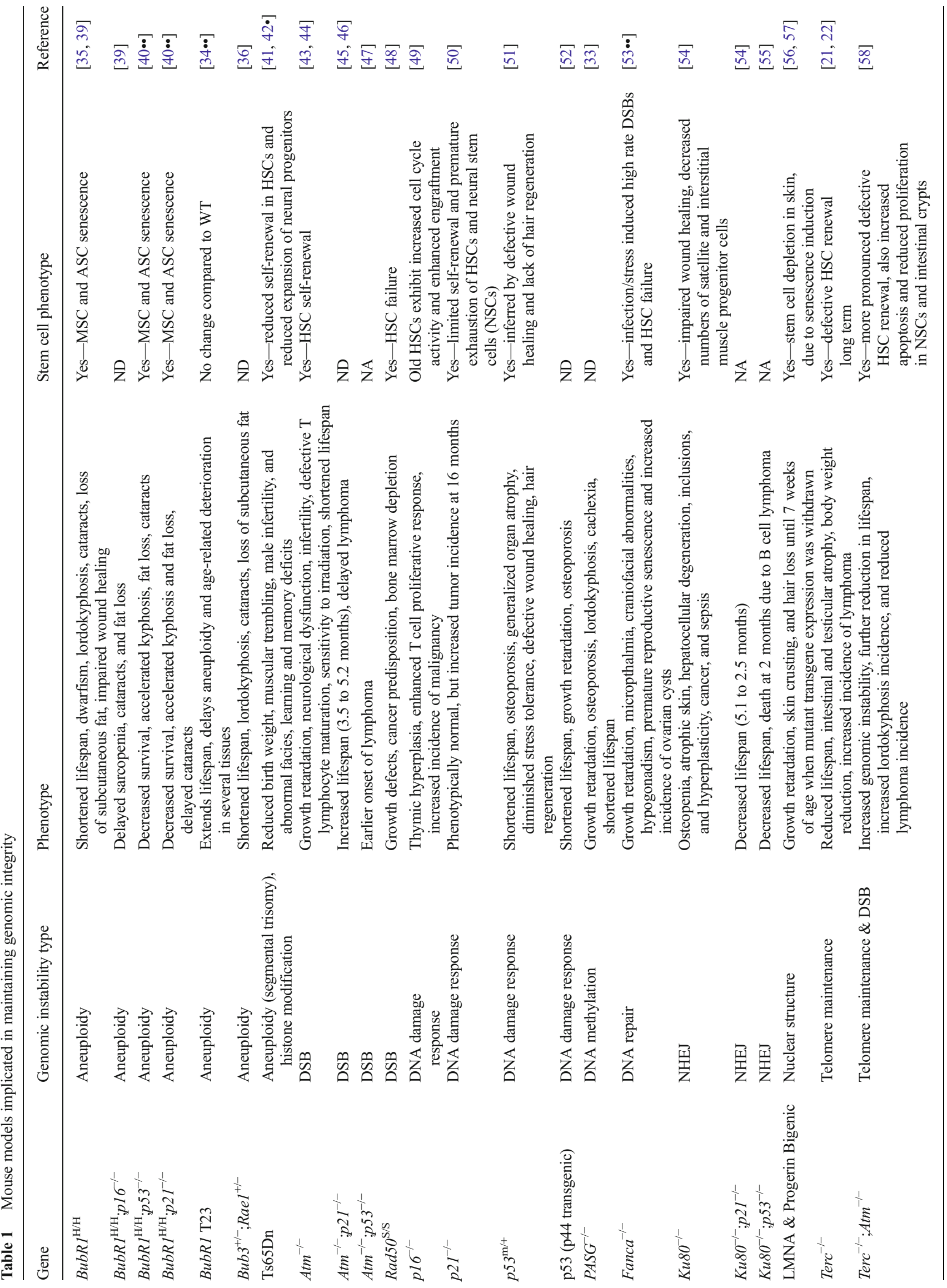


determine the significance of these defects in organismal health.

\section{Role of p53 and CDKi's in DNA Damage Models}

The cyclin-dependent kinase inhibitors (CDKi's) p21 and $\mathrm{p} 16^{\text {Ink4a }}$ are generally considered to enforce transient cell cycle arrest and cellular senescence, respectively [69]. One hypothesis is that p53-induced transcription of $\mathrm{p} 21$ mediates arrest to allow cells time to repair genomic damage prior to replication and division. If this repair fails, senescence or apoptosis is engaged to restrain these damaged and potentially pre-neoplastic cells from transforming. However, combining p21 deficiency with depletion of DNA repair factors leads to variable outcomes for different stem cell types depending on the nature of the DNA damage (Table 1). For example, Terc $^{-1-}$ mice have early aging-associated features along with reduced HSC and intestinal crypt stem cell self-renewal, which is dependent on p53-mediated cell cycle arrest and apoptosis [21]. Ablating Puma, a p53-upregulated modulator of apoptosis, attenuates apoptosis in this model, which leads to an increased lifespan along with improved stem cell function [60]. When cell cycle arrest is prevented by deletion of $\mathrm{p} 21$ genetically $\left(\operatorname{Terc}^{-/} ; p_{21} 1^{-/}\right)$, mice also exhibit improved stem cell selfrenewal, decreased features of progeria, and increased lifespan [59]. $\mathrm{Atm}^{-/}$mice, which suffer from increased double-strand breaks, HSC dysfunction, and osteoporosis, also exhibit a lifespan extension when p21 is simultaneously deleted [70]. However, this improvement is attributed to an increase in apoptosis, which prevents the development of lethal lymphomas [45-47]. An alteration in the onset/delay of progeria in this model has not been reported.

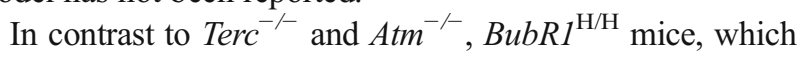
also exhibit accelerated aging phenotypes, have accelerated aging features in adipose and skeletal muscle tissue and a substantially decreased lifespan when p21 is deleted [40••]. Consistent with these results, knockout of p53, the major regulator of $\mathrm{p} 21$, in BubR1 hypomorphic mice produces an earlier onset of age-related dysfunction and earlier death [40••]. Similarly, $K u 80^{-1-}$ mice, which have defective nonhomologous end joining (NHEJ) and profound premature muscle wasting, also exhibit earlier death when either p53 [55] or p21 [54] is depleted. However, the age-related phenotypes were seemingly not impacted. Taken together, these studies demonstrate that the effects of p21 deletion in progeroid mouse models are complex and require a reinterpretation of the role of p21 in response to DNA damage (Fig. 1).

Differences could potentially be explained by variation in the extent or type of DNA damage in these models, leading to alternative cellular fates when transient cell cycle arrest is lost. $\mathrm{Terc}^{-/-}$mice, for example, have a longer lifespan and less DNA damage than $K u 80^{--}$mice and $\mathrm{p} 21$ deletion decreases rates of apoptosis and increases lifespan, whereas the inverse is observed in $\mathrm{Ku} 80^{-/-}$mice $[54,59]$. A strong cell death response can have negative impacts on lifespan, such as stem cell depletion, or positive ones, such as cancer prevention. In light of this, perhaps less damaged $\operatorname{Terc}^{-/-}$cells arrest in a $\mathrm{p} 21-$ dependent manner to facilitate repair, but this repair is not strictly required for cell survival and in fact leads to reduced tissue regeneration. For highly damaged $\mathrm{Ku} 80^{-/-}$cells, however, it appears that p21 arrest (or other functions of p21) is necessary for cell survival, and "relieving" the arrest is counter-productive.

Placing $B u b R I^{\mathrm{H} / \mathrm{H}}$ and $B u b R I^{\mathrm{H} / \mathrm{H}} ; p 21^{-/-}$mice into this structure is difficult, as the underlying "genomic damage" in these models is numerical aneuploidy. Aneuploidy per se is not sufficient to independently drive progeria because other whole chromosome instability mouse models with similar, if not higher, aneuploidy rates do not show accelerated aging $[71,72]$. The observation that $p 16^{\text {Ink4a }}$ deletion improves the progeroid phenotype of $B u b R 1^{\mathrm{H} / \mathrm{H}}$ mice implies that senescence is linked to aging in this model [39]. Clearance of senescent cells by drug-induced apoptosis confirmed these findings [73], indicating that non-autonomous functions of cellular senescence contribute to progeria in BubR1 hypomorphic mice. In contrast to $\mathrm{Ku} 80^{-/-} ; p 21^{-/-}$MEFs, $B u b R 1^{\mathrm{H} / \mathrm{H}} ; p 21^{-/-}$ MEFs show increased $\mathrm{p} 16^{\text {Ink4a }}$ expression [40••], presumably due to loss of p21-mediated cell cycle arrest, which drives damaged cells into senescence rather than repair. Surprisingly, not all prematurely aged $B u b R 1^{\mathrm{H} / \mathrm{H}}$ tissues display this effect. While sarcopenia is accelerated in BubR1 hypomorphic mice with loss of $\mathrm{p} 21$, cataract formation is significantly delayed, which may be the result of increased apoptosis of damaged lens epithelial cells $[40 \bullet \bullet]$. The observation that skeletal muscle progenitor and lens epithelial cells, two distinct cell populations, respond differently to loss of $\mathrm{p} 21$ could be interpreted as unique and preferred responses of stem versus differentiated cells. Intriguingly, p53 deletion also leads to an upregulation of $p 16^{\text {Ink4a }}$ in all $B u b R 1^{\mathrm{H} / \mathrm{H}}$ tissues with early ageassociated changes $[40 \bullet$ ], suggesting that $\mathrm{p} 21$ is mediating tissue and cell type-specific choices between cell survival and death. An alternative possibility, which cannot be excluded, is that $\mathrm{p} 21$ deletion stimulates quiescent stem cells to begin cycling (discussed further below), which could improve tissue function if mild, but lead to stem cell exhaustion if severe. A final complication is that $\mathrm{p} 21$ participates in DNA damage repair directly by binding PCNA to inhibit base excision repair and trans-lesion synthesis [74, 75], in addition to inhibiting DNA replication. However, it remains unclear to what extent the benefits of $\mathrm{p} 21$ deletion arise from enhanced basal DNA repair, altered stem cell quiescence, and directing damaged cells to die or senesce. These contrasting examples illustrate that the response to effector manipulation in vivo cannot necessarily be inferred from in vitro work, perhaps due to differences in the response of relevant cell types to 


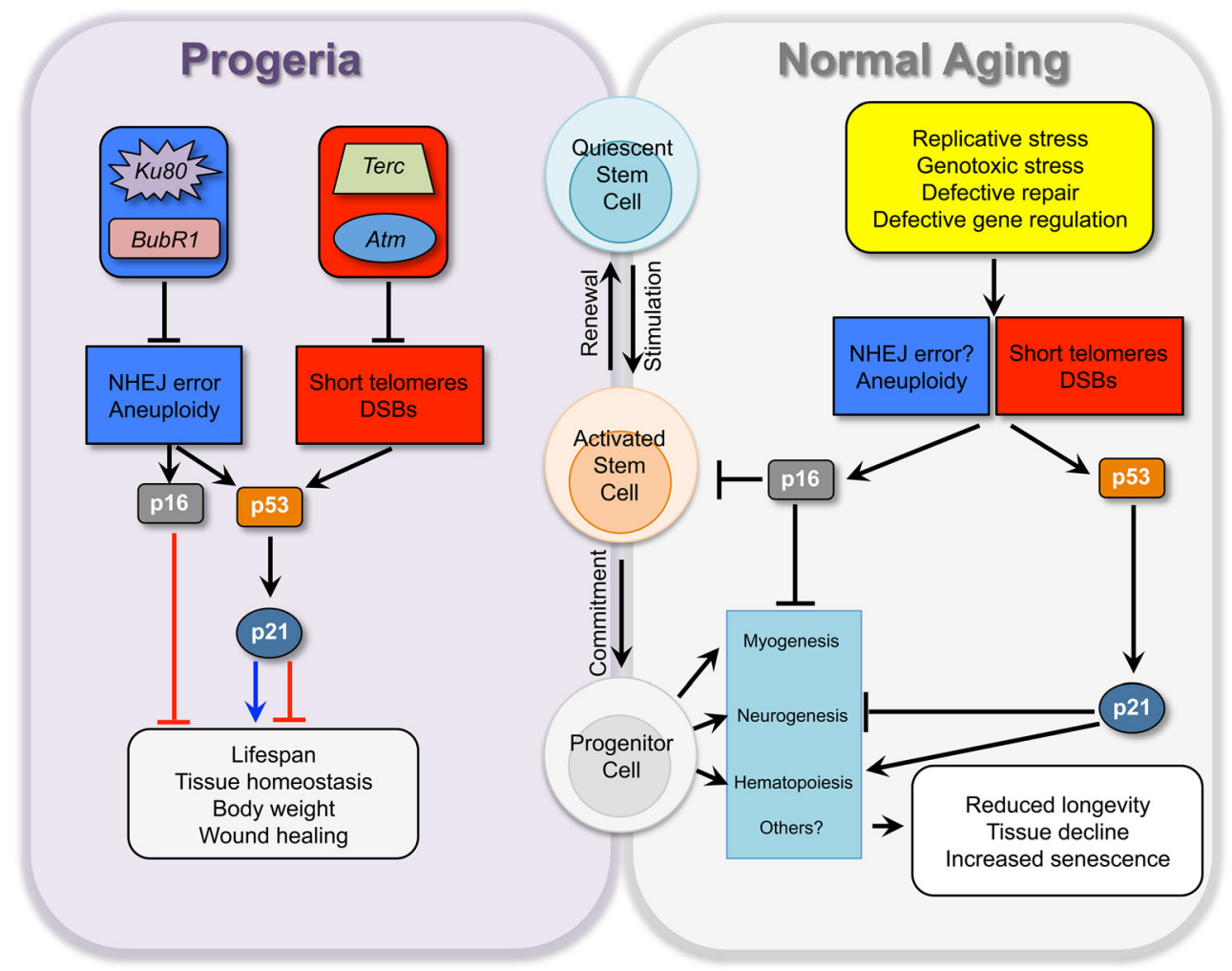

Fig. 1 DNA damage disrupts stem and progenitor cell function in both progeroid models and normal aging. Block background and arrows are of matching colors. In progeria (left panel), knockout of different types of DNA repair proteins leads to stem cell dysfunction syndromes that are modified by effector proteins (p16, p53, and p21) in characteristic ways. For example, $K u 80^{-1-}$ and $B u b R I^{\mathrm{H} / \mathrm{H}}$ tissues show impaired progenitor cell function, which is improved with p 21 activity. In contrast, Terc $^{-1}$ and $\mathrm{Atm}^{-/}$stem cells are lost at the level of stem cell renewal and the overall health of this compartment is aggravated by loss of $\mathrm{p} 21$. The impact of

DNA damage. Finally, the example of $\mathrm{Atm}^{-/-} ; \mathrm{p} 21^{-/-}$is a cautionary example showing that proper interpretation of lifespan differences between progeroid models requires cancer incidence data, particularly when cell death mechanisms are suspected.

\section{Senescence, Effectors, Aging, and the Stem Cell Niche}

Several markers of senescence and cell cycle arrest, including $\mathrm{p} 16^{\mathrm{Ink} 4 \mathrm{a}}$ and $\mathrm{p} 21$, increase with age in multiple tissues [76]. Satellite cells (functioning stem cells of skeletal muscle) of advanced-aged mice undergo $\mathrm{p} 16^{\text {Ink4a }}$-dependent senescence [77•]. Transplantation of these satellite cells that have been "rejuvenated" by decreasing $p 16^{\text {Ink4a }}$ expression via short hairpin RNA (shRNA) significantly improved repair of damaged muscle in geriatric mice [77•]. Consistent with this observation, HSCs that cannot up-regulate $\mathrm{p} 16^{\text {Ink4a }}$ with age exhibit less apoptosis and have improved repopulation specific DNA repair pathways on the stem cell lifecycle is not so well characterized in normal aging as in progerias. However, effector functions are being characterized. For example, just as p21 has differential effects depending on the damage model, in natural aging $\mathrm{p} 21$ restricts hematopoietic progenitor numbers but preserves neurogenesis. The nature of the upstream DNA damage signals driving effector activation is probably mixed and depends on the tissue type, its rate of turnover, and exposure to DNA damaging stresses

capacity [49]. Similar improvements in progenitor cell expansion with age have been observed in neural forebrain progenitors [78] and pancreatic islet stem cells [79] when p16 $6^{\text {Ink4a }}$ has been deleted. Conversely, mice null for Bmi1, which negatively regulates $\mathrm{p} 16^{\mathrm{Ink} 4 \mathrm{a}} / \mathrm{p} 19^{\text {Arf }}$ expression, exhibit premature senescence in HSCs and early death [80, 81]. Enforced expression of $\mathrm{p} 16^{\text {Ink4a }}$ and $\mathrm{p} 19^{\text {Arf }}$ in HSCs promotes senescence and apoptosis, respectively, while deletion of both of these proteins in $\mathrm{Bmil}^{-/-}$mice restores HSC self-renewal [82]. Together, these studies demonstrate that $\mathrm{p} 16^{\text {Ink4a }}$ expression consistently impairs stem cell function (Fig. 1).

The impacts of $\mathrm{p} 21$ in stem cell function during aging are much more complicated, as there are apparently two opposing roles. p21 maintains a quiescent pool of stem cells through cell cycle arrest, which is essential for preserving the stem cell reservoir. Conversely, p21 also may enforce a senescence arrest, which depletes stem cell self-renewal capacity. Therefore, the consequences of $\mathrm{p} 21$ deletion in aging are seemingly dependent on the requirement for a quiescent stem cell reserve. For example, slow-cycling mouse ventral forebrain precursors escape quiescence when p21 is deleted and quickly undergo 
proliferative exhaustion [50]. In more rapidly cycling compartments, such as the bone marrow, aged $p 21^{-/-}$mice do not have defects in HSC numbers or regenerative capacity [83]. Peculiarly, $p 21^{-1-}$ HSCs have improved engraftment and repopulation compared to wild-type HSCs when irradiated. Similar to p $16^{\text {Ink4a }}$-null mice, p53 knockout animals have reduced HSC numbers and engraftment potential with age [84]. Unlike $p 21^{-/-}$HSCs, $p 53^{-/-}$HSCs are dysfunctional even at a young age [85], indicating that a $\mathrm{p} 21$-independent function of $\mathrm{p} 53$ prevents stem cell dysfunction. The observation that reprogramming of damaged, differentiated cells to induced pluripotent stem (iPS) cells is limited by p53 and p21 expression [86] may explain these observations. Perhaps self-renewing asymmetric stem cell division in vivo is regulated by a process similar to reprogramming (i.e., one damaged stem cell giving rise to two progenitor cells rather than a daughter stem cell and progenitor cell). Finally, endothelial progenitor cell function is reduced during both human and mouse aging, partially as a result of senescence driven by the p53-p21 pathway [87, 88]. These results are consistent with a model in which $\mathrm{p} 21$ serves multiple functions in normal stem cell physiology: "time-out" for repair, maintenance of appropriate quiescence, regulating DNA repair, and possibly acting as an anti-apoptotic factor counter-balancing over-active p53. Interestingly, mice with over-active p53 exhibit a variety of premature aging phenotypes [51,52]. With this in mind, it is somewhat surprising that there have been relatively few documented impacts of $\mathrm{p} 21$ deletion on normal organismal aging.

The stem cell niche is a specialized environment in which stem cells give rise to progenitor (transitamplifying) cells and self-renew via asymmetric division [89]. Proper division depends on supporting cells and the extracellular matrix, whereas progenitor cell differentiation occurs by exposure of the maturing cells to a gradient of differentiation factors [90]. Evidence that the stem cell niche loses its pro-stemness properties with age was demonstrated by transplantation of spermatogonial stem cells from old mice. These transplanted cells become rejuvenated and reactivated in the environment of the young testes [91]. The stem cell niche is dysfunctional in both adipose tissue and skeletal muscle of $B u b R 1^{\mathrm{H} / \mathrm{H}}$ mice because both preadipocytes and fibroadipogenic progenitors exhibit higher rates of senescence [40 • $]$. Clearing these senescent progenitor cells by activating apoptosis specifically in p16 ${ }^{\text {Ink4a }}$-expressing cells restores stem cell function [73]. This effect might occur through attenuation of the senescence-associated secretory phenotype (SASP), a characteristic secretome expressed once cells have become senescent [92]. This includes matrix metalloproteinases capable of destroying ECM architecture, as well as TGF- $\beta$ family members [93]. TGF- $\beta$ is known to inhibit proliferation of HSCs by upregulating the CDKi p57 ${ }^{\mathrm{Kip}}$ [94] and has similar effects on satellite cells [95] and preadipocytes [96]. Despite the attractive nature of this hypothesis, whether these mechanisms are at play in aging is unknown.

\section{Conclusion and Outlook}

Many sources of genomic insults result in the accumulation of damage in stem cells with aging that negatively impacts their function. In addition, depletion of stem cells occurs in a number of tissue compartments with aging [97]. Together with the observations that senescence diminishes stem cell function, both directly through senescence of stem cells and indirectly through inhibitory signaling via the SASP, declining tissue repair due to stem cell dysfunction seems almost inevitable. However, a plethora of recent parabiosis studies, where an aged mouse and a young mouse are surgically joined together so that they have a common circulation, have shown that circulating factors present in young blood can have rejuvenating effects on aging in the old animal, including stem cells of the brain [98・•] and skeletal muscle [99••]. Going forward, it will be interesting to determine if these circulating factors result in replacement or replenishment of the defective niches that develop with age, and whether genomic stability of these cells is impacted. These studies have the potential to identify factors capable of promoting stem cell function in the elderly, both on native stem cells and also when given together with stem cell transplants. Furthermore, iPS cells and other transplantable, therapeutic stem cells may acquire aneuploidy and other DNA damage in culture. Understanding the effect of the aged body on the DNA maintenance machinery and whether or not we possess molecules that rejuvenate these processes may influence what we see as acceptable risk for these procedures.

Acknowledgments We apologize sincerely to all those whose work was not cited due to space constraints. We thank Robbyn Weaver, Ryan Naylor, and Jan van Deursen for helpful discussions. This work was supported by the Paul F. Glenn Foundation (D.J.B.), the Ellison Medical Foundation (D.J.B.), and the Robert and Arlene Kogod Center on Aging (D.J.B.).

\section{Compliance with Ethics Guidelines}

Conflict of Interest Cynthia J. Hommerding, Bennett G. Childs, and Darren J. Baker declare that they have no conflict of interest.

Human and Animal Rights and Informed Consent This article does not contain any studies with human or animal subjects performed by any of the authors. 


\section{References}

Papers of particular interest, published recently, have been highlighted as:

- Of importance

•- Of major importance

1. Bartek J, Lukas J. DNA damage checkpoints: from initiation to recovery or adaptation. Curr Opin Cell Biol. 2007;19(2):238-45.

2. Vousden KH, Prives C. Blinded by the light: the growing complexity of p53. Cell. 2009;137(3):413-31.

3. Childs BG et al. Senescence and apoptosis: dueling or complementary cell fates? EMBO Rep. 2014;15(11):1139-53.

4. Moskalev AA et al. The role of DNA damage and repair in aging through the prism of Koch-like criteria. Ageing Res Rev. 2013;12(2):661-84.

5. Burtner CR, Kennedy BK. Progeria syndromes and ageing: what is the connection? Nat Rev Mol Cell Biol. 2010;11(8):567-78.

6. Negrini S, Gorgoulis VG, Halazonetis TD. Genomic instabilityan evolving hallmark of cancer. Nat Rev Mol Cell Biol. 2010;11(3): $220-8$.

7. Weissman IL. Stem cells: units of development, units of regeneration, and units in evolution. Cell. 2000;100(1):157-68.

8. Li L, Clevers H. Coexistence of quiescent and active adult stem cells in mammals. Science. 2010;327(5965):542-5.

9. Burkhalter MD, Rudolph KL, Sperka T. Genome instability of ageing stem cells-induction and defence mechanisms. Ageing Res Rev. 2015.

10. Mimeault M, Batra SK. Recent insights into the molecular mechanisms involved in aging and the malignant transformation of adult stem/progenitor cells and their therapeutic implications. Ageing Res Rev. 2009;8(2):94-112.

11. Blasco MAA. Telomeres in cancer and aging: lessons from the mouse. Cancer Lett. 2003;194(2):183-8.

12. de Lange T. Protection of mammalian telomeres. Oncogene. 2002;21(4):532-40.

13. Chiu CP et al. Differential expression of telomerase activity in hematopoietic progenitors from adult human bone marrow. Stem Cells. 1996;14(2):239-48.

14. Wright WE et al. Telomerase activity in human germline and embryonic tissues and cells. Dev Genet. 1996;18(2):173-9.

15. Flores I et al. The longest telomeres: a general signature of adult stem cell compartments. Genes Dev. 2008;22(5):654-67.

16. Vaziri $\mathrm{H}$ et al. Evidence for a mitotic clock in human hematopoietic stem cells: loss of telomeric DNA with age. Proc Natl Acad Sci U S A. 1994;91(21):9857-60.

17. Hayflick L. The limited in vitro lifetime of human diploid cell strains. Exp Cell Res. 1965;37:614-36.

18. Harley CB, Futcher AB, Greider CW. Telomeres shorten during ageing of human fibroblasts. Nature. 1990;345(6274):458-60.

19. Hayflick L, Moorhead PS. The serial cultivation of human diploid cell strains. Exp Cell Res. 1961;25:585-621.

20. Armanios M, Blackburn EH. The telomere syndromes. Nat Rev Genet. 2012;13(10):693-704.

21. Espejel $\mathrm{S}$ et al. Impact of telomerase ablation on organismal viability, aging, and tumorigenesis in mice lacking the DNA repair proteins PARP-1, Ku86, or DNA-PKcs. J Cell Biol. 2004;167(4):62738.

22. Lee HW et al. Essential role of mouse telomerase in highly proliferative organs. Nature. 1998;392(6676):569-74.

23. Vijg J, Suh Y. Genome instability and aging. Annu Rev Physiol. 2013;75:645-68

24. Hsieh JC et al. Large chromosome deletions, duplications, and gene conversion events accumulate with age in normal human colon crypts. Aging Cell. 2013;12(2):269-79. This study demonstrated that aged colonic crypts exhibit a large amount of genomic instability with advanced age, which was independent of indicators of tumorigenesis.

25. Stracker TH et al. The ATM signaling network in development and disease. Front Genet. 2013;4:37.

26. Jaenisch R, Bird A. Epigenetic regulation of gene expression: how the genome integrates intrinsic and environmental signals. Nat Genet. 2003;33(Suppl):245-54.

27. Dang $\mathrm{W}$ et al. Histone $\mathrm{H} 4$ lysine 16 acetylation regulates cellular lifespan. Nature. 2009;459(7248):802-7.

28. Sarg B et al. Postsynthetic trimethylation of histone $\mathrm{H} 4$ at lysine 20 in mammalian tissues is associated with aging. $\mathrm{J}$ Biol Chem. 2002;277(42):39195-201.

29. Greer EL et al. Members of the H3K4 trimethylation complex regulate lifespan in a germline-dependent manner in C. elegans. Nature. 2010;466(7304):383-7.

30. Scaffidi P, Misteli T. Reversal of the cellular phenotype in the premature aging disease Hutchinson-Gilford progeria syndrome. Nat Med. 2005;11(4):440-5.

31. Maures TJ et al. The H3K27 demethylase UTX-1 regulates C. elegans lifespan in a germline-independent, insulin-dependent manner. Aging Cell. 2011;10(6):980-90.

32. Zampieri M, et al. Reconfiguration of DNA methylation in aging. Mech Ageing Dev. 2015.

33. Sun LQ et al. Growth retardation and premature aging phenotypes in mice with disruption of the SNF2-like gene. PASG Genes Dev. 2004;18(9):1035-46.

34.• Baker DJ et al. Increased expression of BubR1 protects against aneuploidy and cancer and extends healthy lifespan. Nat Cell Biol. 2013;15(1):96-102. Enforced expression of BubR1 throughout life led to improved chromosome segregation efficiency, improved healthspan and extended lifespan.

35. Baker DJ et al. BubR1 insufficiency causes early onset of agingassociated phenotypes and infertility in mice. Nat Genet. 2004;36(7):744-9.

36. Baker DJ et al. Early aging-associated phenotypes in Bub3/Rae1 haploinsufficient mice. J Cell Biol. 2006;172(4):529-40.

37. Hanks $\mathrm{S}$ et al. Constitutional aneuploidy and cancer predisposition caused by biallelic mutations in BUB1B. Nat Genet. 2004;36(11): 1159-61.

38. Garcia-Castillo $\mathrm{H}$ et al. Clinical and genetic heterogeneity in patients with mosaic variegated aneuploidy: delineation of clinical subtypes. Am J Med Genet A. 2008;146A(13):1687-95.

39. Baker DJ et al. Opposing roles for p16Ink4a and p19Arf in senescence and ageing caused by BubR1 insufficiency. Nat Cell Biol. 2008;10(7):825-36.

40.• Baker DJ, Weaver RL, van Deursen JM. p21 both attenuates and drives senescence and aging in BubR1 progeroid mice. Cell Rep. 2013;3(4):1164-74. In this manuscript, the cell types responsible for senescence of BubR1 hypomorphic mice are identified as progenitor cells of adipose tissue and skeletal muscle. Depletion of p21 in BubR1 mutant mice decreases lifespan, accelerates kyphosis and fat loss, but delays cataract formation.

41. Reeves RH et al. A mouse model for Down syndrome exhibits learning and behaviour deficits. Nat Genet. 1995;11(2):177-84.

42. Adorno $\mathrm{M}$ et al. Usp16 contributes to somatic stem-cell defects in Down's syndrome. Nature. 2013;501(7467):380-4. Copy number variation at a specific locus, Usp16, rather than a global response to aneuploidy, promotes disease phenotypes in this mouse model of DS.

43. Barlow $\mathrm{C}$ et al. Atm-deficient mice: a paradigm of ataxia telangiectasia. Cell. 1996;86(1):159-71.

44. Ito $\mathrm{K}$ et al. Regulation of oxidative stress by ATM is required for self-renewal of haematopoietic stem cells. Nature. 2004;431(7011): 997-1002. 
45. Shen $\mathrm{KC}$ et al. ATM and $\mathrm{p} 21$ cooperate to suppress aneuploidy and subsequent tumor development. Cancer Res. 2005;65(19):874753.

46. Wang YA, Elson A, Leder P. Loss of p21 increases sensitivity to ionizing radiation and delays the onset of lymphoma in atmdeficient mice. Proc Natl Acad Sci U S A. 1997;94(26):14590-5.

47. $\mathrm{Xu} \mathrm{Y}$ et al. Involvement of p53 and p21 in cellular defects and tumorigenesis in Atm-/- mice. Mol Cell Biol. 1998;18(7):438590.

48. Bender $\mathrm{CF}$ et al. Cancer predisposition and hematopoietic failure in Rad50(S/S) mice. Genes Dev. 2002;16(17):2237-51.

49. Janzen $V$ et al. Stem-cell ageing modified by the cyclin-dependent kinase inhibitor p16INK4a. Nature. 2006;443(7110):421-6.

50. Kippin TE, Martens DJ, van der Kooy D. p21 loss compromises the relative quiescence of forebrain stem cell proliferation leading to exhaustion of their proliferation capacity. Genes Dev. 2005;19(6): 756-67.

51. Tyner SD et al. p53 mutant mice that display early ageingassociated phenotypes. Nature. 2002;415(6867):45-53.

52. Maier B et al. Modulation of mammalian life span by the short isoform of p53. Genes Dev. 2004;18(3):306-19.

53.• Walter D, et al. Exit from dormancy provokes DNA-damageinduced attrition in haematopoietic stem cells. Nature. 2015. This study shows that repeated activation of normal HSCs by exposure to physiological stress, such as infection or chronic blood loss, induced DNA damage and provoked attrition. Furthermore, complete HSC failure was observed in Faconi anemia mutants.

54. Zhao B et al. Cellular senescence and organismal ageing in the absence of p21(CIP1/WAF1) in ku80(-/-) mice. EMBO Rep. 2009;10(1):71-8.

55. Lim DS et al. Analysis of ku80-mutant mice and cells with deficient levels of p53. Mol Cell Biol. 2000;20(11):3772-80.

56. Sagelius $\mathrm{H}$ et al. Targeted transgenic expression of the mutation causing Hutchinson-Gilford progeria syndrome leads to proliferative and degenerative epidermal disease. J Cell Sci. 2008;121(Pt 7): 969-78.

57. Rosengardten $\mathrm{Y}$ et al. Stem cell depletion in Hutchinson-Gilford progeria syndrome. Aging Cell. 2011;10(6):1011-20.

58. Wong KK et al. Telomere dysfunction and Atm deficiency compromises organ homeostasis and accelerates ageing. Nature. 2003;421(6923):643-8.

59. Choudhury AR et al. Cdkn1a deletion improves stem cell function and lifespan of mice with dysfunctional telomeres without accelerating cancer formation. Nat Genet. 2007;39(1):99-105.

60. Sperka $\mathrm{T}$ et al. Puma and $\mathrm{p} 21$ represent cooperating checkpoints limiting self-renewal and chromosomal instability of somatic stem cells in response to telomere dysfunction. Nat Cell Biol. 2012;14(1):73-9.

61. Tomas-Loba A et al. Telomerase reverse transcriptase delays aging in cancer-resistant mice. Cell. 2008;135(4):609-22.

62. Lee JH, Paull TT. Activation and regulation of ATM kinase activity in response to DNA double-strand breaks. Oncogene. 2007;26(56): 7741-8.

63. Meyn MS. Ataxia-telangiectasia and cellular responses to DNA damage. Cancer Res. 1995;55(24):5991-6001.

64.• Beerman I et al. Quiescent hematopoietic stem cells accumulate DNA damage during aging that is repaired upon entry into cell cycle. Cell Stem Cell. 2014;15(1):37-50. Mouse HSCs accumulate DSBs during aging, however, upon reentry into the cell cycle, repair pathways are upregulated and these damages are rapidly repaired.

65.• Flach J et al. Replication stress is a potent driver of functional decline in ageing haematopoietic stem cells. Nature. 2014;512(7513):198-202. HSCs in old mice exhibit heightened replication stress associated with cell cycle defects and chromosome gaps or breaks due to decreased expression of mini-chromosome maintenance (MCM) components and altered dynamics of DNA replication forks.

66. Eriksson $\mathrm{M}$ et al. Recurrent de novo point mutations in lamin $\mathrm{A}$ cause Hutchinson-Gilford progeria syndrome. Nature. 2003;423(6937):293-8.

67. Liu B et al. Stem and progenitor cell dysfunction in human trisomies. EMBO Rep. 2015;16(1):44-62.

68. Rossi DJ et al. Hematopoietic stem cell quiescence attenuates DNA damage response and permits DNA damage accumulation during aging. Cell Cycle. 2007;6(19):2371-6.

69. van Deursen JM. The role of senescent cells in ageing. Nature. 2014;509(7501):439-46.

70. Rasheed $\mathrm{N}$ et al. Atm-deficient mice: an osteoporosis model with defective osteoblast differentiation and increased osteoclastogenesis. Hum Mol Genet. 2006;15(12):1938-48.

71. Dawlaty $\mathrm{MM}$ et al. Resolution of sister centromeres requires RanBP2-mediated SUMOylation of topoisomerase IIalpha. Cell. 2008;133(1):103-15.

72. Ricke RM, Jeganathan KB, van Deursen JM. Bub1 overexpression induces aneuploidy and tumor formation through Aurora B kinase hyperactivation. J Cell Biol. 2011;193(6):1049-64.

73. Baker DJ et al. Clearance of p16Ink4a-positive senescent cells delays ageing-associated disorders. Nature. 2011;479(7372):232-6.

74. Tom S et al. Regulatory roles of $\mathrm{p} 21$ and apurinic/apyrimidinic endonuclease 1 in base excision repair. J Biol Chem. 2001;276(52):48781-9.

75. Soria $\mathrm{G}$ et al. p21 differentially regulates DNA replication and DNA-repair-associated processes after UV irradiation. J Cell Sci. 2008;121(Pt 19):3271-82.

76. Krishnamurthy $\mathrm{J}$ et al. Ink4a/Arf expression is a biomarker of aging. J Clin Invest. 2004;114(9):1299-307.

77. Sousa-Victor P et al. Geriatric muscle stem cells switch reversible quiescence into senescence. Nature. 2014;506(7488):316-21. Satellite cells from aged mice undergo p16-dependent cellular senescence. Reduction of p16 using shRNA prevents this engagement and results in functional restoration of these aged cells.

78. Molofsky AV et al. Increasing p16INK4a expression decreases forebrain progenitors and neurogenesis during ageing. Nature. 2006;443(7110):448-52.

79. Krishnamurthy J et al. p16INK4a induces an age-dependent decline in islet regenerative potential. Nature. 2006;443(7110):453-7.

80. Park IK et al. Bmi-1 is required for maintenance of adult selfrenewing haematopoietic stem cells. Nature. 2003;423(6937): $302-5$.

81. Lessard J, Sauvageau G. Bmi-1 determines the proliferative capacity of normal and leukaemic stem cells. Nature. 2003;423(6937): 255-60.

82. Oguro $\mathrm{H}$ et al. Differential impact of Ink4a and Arf on hematopoietic stem cells and their bone marrow microenvironment in Bmi1deficient mice. J Exp Med. 2006;203(10):2247-53.

83. van Os $\mathrm{R}$ et al. A limited role for $\mathrm{p} 21 \mathrm{Cip} 1 / \mathrm{Waf} 1$ in maintaining normal hematopoietic stem cell functioning. Stem Cells. 2007;25(4):836-43.

84. Dumble $\mathrm{M}$ et al. The impact of altered $\mathrm{p} 53$ dosage on hematopoietic stem cell dynamics during aging. Blood. 2007;109(4):1736-42.

85. TeKippe M, Harrison DE, Chen J. Expansion of hematopoietic stem cell phenotype and activity in Trp53-null mice. Exp Hematol. 2003;31(6):521-7.

86. Marion RM et al. A p53-mediated DNA damage response limits reprogramming to ensure iPS cell genomic integrity. Nature. 2009;460(7259):1149-53.

87. Rosso A et al. p53 mediates the accelerated onset of senescence of endothelial progenitor cells in diabetes. J Biol Chem. 2006;281(7): 4339-47. 
88. Satoh $\mathrm{M}$ et al. Association between oxidative DNA damage and telomere shortening in circulating endothelial progenitor cells obtained from metabolic syndrome patients with coronary artery disease. Atherosclerosis. 2008;198(2):347-53.

89. Calvi LM, Link DC. Cellular complexity of the bone marrow hematopoietic stem cell niche. Calcif Tissue Int. 2014;94(1):112-24.

90. Gregorieff A et al. Expression pattern of Wnt signaling components in the adult intestine. Gastroenterology. 2005;129(2):626-38.

91. Ryu BY et al. Effects of aging and niche microenvironment on spermatogonial stem cell self-renewal. Stem Cells. 2006;24(6): 1505-11.

92. Coppe JP et al. Senescence-associated secretory phenotypes reveal cell-nonautonomous functions of oncogenic RAS and the p53 tumor suppressor. PLoS Biol. 2008;6(12):2853-68.

93. Coppe JP et al. A human-like senescence-associated secretory phenotype is conserved in mouse cells dependent on physiological oxygen. PLoS One. 2010;5(2), e9188.

94. Scandura JM et al. Transforming growth factor beta-induced cell cycle arrest of human hematopoietic cells requires p57KIP2 upregulation. Proc Natl Acad Sci U S A. 2004;101(42):15231-6.
95. Allen RE, Boxhorn LK. Regulation of skeletal muscle satellite cell proliferation and differentiation by transforming growth factor-beta, insulin-like growth factor I, and fibroblast growth factor. J Cell Physiol. 1989;138(2):311-5.

96. Shin SM et al. Dexamethasone reverses TGF-beta-mediated inhibition of primary rat preadipocyte differentiation. FEBS Lett. 2003;543(1-3):25-30.

97. Liu L, Rando TA. Manifestations and mechanisms of stem cell aging. J Cell Biol. 2011;193(2):257-66.

98.• Katsimpardi L et al. Vascular and neurogenic rejuvenation of the aging mouse brain by young systemic factors. Science. 2014;344(6184):630-4. Parabiosis studies of young and old mice sharing a common circulation reveal a rejuvenating effect on neural stems cells of aged mice.

99.• Sinha $\mathrm{M}$ et al. Restoring systemic GDF11 levels reverses agerelated dysfunction in mouse skeletal muscle. Science. 2014;344(6184):649-52. This study identified a circulating factor from young mice that has rejuvenating effects on aged mice through parabiosis studies. Supplementation of this single factor had beneficial impacts on skeletal muscle of aged mice. 